\title{
GRAPH ORIENTATION SATISFYING THAT ALL NODES IN A GIVEN SUBSET HAVE IN- OR OUT-DEGREE 0, AND EVERY OTHER NODE POSITIVE INDEGREE
}

\author{
THINH D. NGUYEN
}

\begin{abstract}
We show that it is hard to orient a digraph such that all nodes in a given subset have in- or out-degree 0 , and every other node positive indegree.
\end{abstract}

\section{Definiton And Claim}

In a directed graph, the indegree of a node is the number of incoming edges and the outdegree is the number of outgoing edges. Consider the following problem. Given an undirected graph $G=(V, E)$ and a designated subset $C \subseteq V(G)$, decide whether it is possible to orient all edges of $G$ so that every node in $C$ has indegree 0 or outdegree 0 , and every other node in $G$ has indegree at least 1 ?

Our problem is now formally defined:

Definition 1. InOutDegOrientation:

Input: An undirected graph $G=(V, E)$ and a set $C \subseteq V(G)$

Output: YES if $G$ has an orientation according to which every node in $C$ has either indegree or outdegree equals to 0 and every node in $V \backslash C$ has positive indegree, otherwise No

Claim 2. We have that 3 Sat $\leq_{p}$ InOUtDegOrientation

[1] demonstrated the hardness of 3SAT. So, our claim above implies hardness for INOUtDegOrientation.

\section{Proof of the Claim 2}

Proof. Our problem is NP-complete by a reduction from 3SAT.

For each literal either positive or negative, create a vertex for it.

Set $C$ to be the set of literals themselves.

For each pair of literals of the same variable $x_{i}$, namely $x_{i}$ and $\neg x_{i}$, connect them by an edge. This ensures that whenever $x_{i}$ is assigned to TRUE (all outgoing arcs) then $\neg x_{i}$ is assigned to FALSE (all incoming arcs), and vice versa whenever $\neg x_{i}$ is assigned to TRUE (all outgoing) then $x_{i}$ is assigned to FALSE (all incoming). Hence, this makes sure the assignment is consistent.

Now, we take care of the clauses.

For each clause, create for it a new vertex. Connect this new clause-vertex to its 3 literal-vertices in $C$. And so, we are done.

A satisfying assignment exists if and only if the produced undirected graph can be oriented such that each vertex in $C$ is either a source vertex, i.e. TRUE with all

Key words and phrases. digraph, orientation, in-degree, out-degree.

Perebor. 
outgoing arcs, or a sink vertex, i.e. FALSE with all incoming arcs. A clause-vertex needs to have at least one satisfying literal which is witnessed by the direction of an incoming arc. The claim follows.

\section{Conclusion}

Garey and Johnson [1] shape their theory based on previous works of Cook, Levin and Karp. Johnson [2] moves on with the guide to this theory. As long as we study a mathematical conjecture, we should encourage ourselves to have enough labour hours on popular mathematics books like these. Then, reading some articles on theory of computing like [3] is a good practice. Only after that, could we think of the ultimate final for all mathematical sciences.

\section{REFERENCES}

1. Michael R. Garey, David S. Johnson, Computers and Intractability: A Guide to the Theory of NP-Completeness

2. David S. Johnson, The NP-Completeness Column: An Ongoing Guide

3. Phan Dinh Dieu, Le Cong Thanh, Le Tuan Hoa, Average Polynomial Time Complexity of Some NP-Complete Problems, Theor. Comput. Sci. 46(3): pp.219-237 (1986)

Email address: kosmofarmer@yandex.com 